\title{
The relationship of sleep duration and insomnia to risk of hypertension incidence: a meta-analysis of prospective cohort studies
}

\author{
Lin Meng ${ }^{1}$, Yang Zheng ${ }^{1}$ and Rutai $\mathrm{Hui}^{2}$
}

To assess whether habitual sleep duration or insomnia increase the incidence of hypertension. PubMed, EMBASE and Cochrane were searched without language restriction. Prospective cohort studies of adults with at least a 1-year follow-up duration were included. Habitual sleep duration or symptoms of insomnia were assessed as baseline exposure, and the outcome was incidence of hypertension. Subgroup, meta-regression and sensitivity analyses were conducted to assess heterogeneity, and Egger's test was used to assess publication bias. Eleven studies (17 cohorts) were included. Short sleep duration, sleep continuity disturbance (SCD), early-morning awakening (EMA) and combined symptoms of insomnia increased the risk of hypertension incidence (the relative risks (95\% confidence intervals) were $1.21(1.05-1.40)$ for short sleep duration, 1.20 (1.06-1.36) for SCD, 1.14 (1.07-1.20) for EMA and 1.05 (1.01-1.08) for combined insomnia symptoms). Less evidence exists to support conclusions about the association between long sleep duration or difficulty falling asleep (DFA) and hypertension incidence. No obvious heterogeneity or publication biases were found. Our meta-analysis demonstrates that short sleep duration and single/combined symptoms of insomnia (except DFA) are associated with an increased risk of hypertension incidence. It is important to consider sleep duration and insomnia during hypertension prevention and treatment. More laboratory studies on potential mechanisms and prospective observational studies with objective measures of sleep are needed. Hypertension Research (2013) 36, 985-995; doi:10.1038/hr.2013.70; published online 5 September 2013

Keywords: insomnia; meta-analysis; sleep; sleep disorder

\section{INTRODUCTION}

Sleep is an ancestral and primitive behavior that is usually manifested as a reversible physiological state characterized by reduced motility and reduced responsiveness to sensory stimuli. ${ }^{1}$ Rats deprived of sleep will exhibit symptoms such as weight loss, increased food intake, decreased body temperature and even death. ${ }^{2}$ Sleep is therefore considered necessary for life. ${ }^{3}$ Human sleep is composed of rapid eye movement sleep and stages 1-4 of non-rapid eye movement sleep; stages 3 and 4 are collectively called slow-wave sleep or deep sleep. These sleep stages are particularly important for general biological restoration. ${ }^{4}$ However, although sleep is a tightly controlled process orchestrated by multiple regulatory systems, sleep disorders do occur. Some sleep disorders result from disruptions in sleep circuitry, some are secondary to other conditions and others result from modern lifestyles. ${ }^{3}$ Insomnia, a common sleep disorder characterized by $24-\mathrm{h}$ hyperarousal, ${ }^{5}$ is defined as a complaint of prolonged sleep latency, difficulties in maintaining sleep and nonrefreshing or poor sleep coupled with impairments of daytime functioning, including reduced alertness, fatigue, exhaustion and dysphoria. ${ }^{5}$ Insomnia occurs acutely in association with predisposing and precipitating factors (for example, psychosocial stressors) and chronically in association with perpetuating factors (for example, extending the time spent in bed). ${ }^{6}$ The behavioral perspective is extended by explicitly allowing the possibility that conditioned arousal may act as a perpetuating factor. ${ }^{5}$ Sleep loss is a substantial problem in modern society, and it may result from either a voluntary restriction of time spent in bed or from a sleep disorder, such as insomnia or obstructive sleep apnea. ${ }^{7}$ In addition, short sleep, generally defined as a habitual sleep time of $6 \mathrm{~h}$ or less, ${ }^{8}$ has been associated with self-reported 'sleeping disorders or insomnia,' 'difficulties in getting sleep without sleeping medicines,' 'overexertion or exhaustion' and 'being awake during the night.' ${ }^{\text {It }}$ should also be noted that the population of short sleepers usually includes both people who purposefully curtail their sleep and those with insomnia who want to sleep more but are not able to do so. ${ }^{8}$

\footnotetext{
${ }^{1}$ Department of Cardiology, Bethune First Hospital of Jilin University, Changchun, China and ${ }^{2}$ Sino-German Laboratory for Molecular Medicine, The State Key Laboratory of Cardiovascular Disease, FuWai Hospital, National Center for Cardiovascular Disease, Chinese Academy of Medical Sciences and Peking Union Medical College, Beijing, China Correspondence: Dr Y Zheng, Department of Cardiology Bethune First Hospital of Jilin University, 71Xinmin Street, Changchun, Jilin Province 130021, China. E-mail: zhengyangls@163.com

or Dr R Hui, Sino-German Laboratory for Molecular Medicine, The State Key Laboratory of Cardiovascular Disease, FuWai Hospital, National Center for Cardiovascular Disease, Chinese Academy of Medical Sciences and Peking Union Medical College, 167 Beilishilu, Beijing 100037, China. E-mail: huirutai@yeah.net
}

Received 26 December 2012; revised 3 March 2013; accepted 10 April 2013; published online 5 September 2013 
Sleep curtailment and disturbances may have deleterious effects on metabolism, endocrinology and immunology, and may eventually increase the risk of disease, including hypertension. ${ }^{9-14}$

Over the last few decades, short-term, acute laboratory sleep deprivation studies have found that blood pressure (BP) and sympathetic nervous system activity increased significantly after sleep restriction. ${ }^{15,16}$ Further, an intervention study showed that melatonin (a hormone that is secreted at night and that may affect many physiological functions, including sleep promotion) is effective for reducing $\mathrm{BP} .{ }^{17}$

Cross-sectional observational studies, in general, support a relationship between short sleep duration or insomnia and higher BP. ${ }^{18-20}$ In addition, some studies have found an association between long sleep duration and prevalent hypertension. ${ }^{19,21}$ The causality of the association between the long-term effects of sleep duration or insomnia and hypertension has been examined in prospective population studies. Several studies indicate that short sleep and insomnia increase the risk of hypertension. However, the conclusions are inconsistent, probably because of differences in the sample types and sizes, the duration of follow-up, the size of the effects and some other variations in study design and exposure/outcome assessment. For example, the effects of insomnia on hypertension or higher BP seem to be influenced by age, ${ }^{22,23}$ different insomnia symptoms ${ }^{22}$ and insomnia severity or duration. ${ }^{24-26}$

Meta-analysis might help to resolve this inconsistency. A recent meta-analysis ${ }^{27}$ assessed the association between sleep duration and the risk of hypertension using literature research updated on 28 October 2011. However, to our knowledge, two prospective cohort studies on the association between sleep duration and hypertension have been published since then; ${ }^{24,28}$ furthermore, no meta-analysis has yet been conducted to address the association between insomnia and hypertension incidence. Therefore, the goal of the present metaanalysis was to quantitatively estimate whether sleep duration or insomnia increase the risk of hypertension incidence, using the most recent data.

\section{METHODS}

We aimed to detect the causal association between sleep duration or insomnia symptoms and incidence of hypertension. Cross-sectional studies could not reflect such a causal direction because of inherent flaws in that design. Therefore, the meta-analysis included only data from prospective cohort studies that reported the association between habitual sleep duration or insomnia and hypertension incidence in normotensive adults and that were published before 4 September 2012. Study identification methods, eligibility criteria, extraction and synthesis of information and results reporting were predetermined. $^{29}$

\section{Literature search}

The electronic databases PubMed, EMBASE and Cochrane were searched using the following terms: '[insomnia or sleep* or (sleep disorder) or (sleep disturbance) and (hyperten* or (high BP)] and (cohort study)' limited to 'human' study with no language restriction. Both published and unpublished data were included. Furthermore, studies from literature references and review articles were searched for additional studies that met the inclusion criteria.

\section{Study selection}

We (LM and YZ) independently identified studies that met the following predetermined inclusion criteria: original article, prospective design, assessment of habitual sleep duration or insomnia symptoms as baseline exposure, hypertension incidence as an outcome (hypertension was defined as a repeatedly elevated systolic BP exceeding 140 and/or diastolic BP over $90 \mathrm{~mm} \mathrm{Hg}$ determined in interview, use of antihypertensive medications or self-reported or recorded diagnosed hypertension), follow-up of at least 1 year and adult population. No sample size restriction was applied.

Studies with cross-sectional or case-control designs were excluded. Considering the special nature of pregnancy, studies that focused on pregnant women were excluded. In cases of multiple reports on the same data set, only the report with the most detailed information for both exposure and outcome was included, with a preference for larger sample sizes and longer follow-up periods. If necessary, the corresponding authors of the eligible studies were contacted with written requests for additional information. The disagreements between two raters were resolved by reaching a consensus among the three investigators involved in the meta-analysis during the selection procedure.

\section{Data extraction}

The following information was abstracted and evaluated independently by two investigators (LM and YZ): first author's surname, year of publication, country of origin of the population studied, follow-up duration, sample size and characteristics (age, sex and race), reference category, definition of 'short' and 'long' sleep and for symptoms of insomnia, sleep/insomnia assessment, outcome assessment, adjusted effect estimate (EE) and 95\% confidence intervals and the variables used in multivariable analyses. If adjusted EEs were given in different levels of adjustment, the most heavily adjusted level was selected.

\section{Definition of sleep duration and insomnia}

Sleep duration was assessed by self-reported habitual sleep duration at night or for both night and daytime sleep using questionnaires (one study measured sleep duration using wrist actigraphy ${ }^{18}$ and one using polysomnography ${ }^{24}$ ). Short sleep was defined as $<5^{26,28,30,31}$ or $<6 \mathrm{~h}^{24}$ per night. Long sleep was defined as $>7,,^{28}>8,{ }^{32} \geqslant 9,26,30$ or $10-15 \mathrm{~h}^{31}$ per night. Symptoms of insomnia were assessed using questionnaires (producing scores) or surveys, except in one study that used wrist actigraphy. ${ }^{18}$ Insomnia was categorized as difficulty falling asleep (DFA) or difficulty initiating sleep, ${ }^{22,23,25,33}$ sleep continuity disturbance (SCD) or difficulty maintaining sleep, ${ }^{18,22,23,25,33}$ early-morning awakening (EMA) ${ }^{23,33}$ and the combination of all symptoms, ${ }^{22-24,26,33}$ with the consideration of persistency of insomnia. In one study in which sleep maintenance was measured as a continuous variable using wrist actigraphy, ${ }^{18}$ subjects were stratified into the SCD/difficulty maintaining sleep group, and the reciprocal values of the original EE and the corresponding 95\% confidence intervals (per 10\% maintenance) were recorded as the effect of SCD on the risk of hypertension incidence. Studies in which insomnia ${ }^{26,33}$ was shown as a continuous variable using sleep-quality scores were classified as having examined a combination of all symptoms. The combination of all symptoms of insomnia was defined as DFA and SCD plus morning sleepiness, ${ }^{22}$ DFA and SCD plus EMA, ${ }^{23}$ any symptom conjugation plus persistency of insomnia, ${ }^{24}$ or continuous sleep scores. ${ }^{26,33}$ Persistency of insomnia was defined as a duration of insomnia symptoms $>4$ years, ${ }^{25}>1$ year ${ }^{24}$ or as frequency variations (from never to always). ${ }^{26}$

\section{Quality assessment}

Two investigators (LM and $\mathrm{RH}$ ) performed the quality assessment of the metaanalysis using the Newcastle-Ottawa scale for cohort studies. ${ }^{34}$ This scale allocates a maximum of nine points for quality of selection, comparability, exposure and outcome of study participants. The two authors discussed the implementation of this assessment tool and agreed on a method of implementation before they independently assessed the studies. The level of agreement between the two reviewers was calculated by another investigator (YZ).

\section{Quantitative data synthesis}

Data from studies on sleep duration and data from studies on insomnia were pooled separately. Hazard ratios, relative risks and odds ratios were combined from multivariable analyses.

We used the random-effects model to quantify the pooled EEs and tested heterogeneity using the $I^{2}$ test and the $\chi^{2}$-test to indicate the proportion of total variance explained by heterogeneity. We also performed meta-regression using a random-effects model. ${ }^{35}$ 


\section{Subgroup analysis}

Prespecified subgroup comparisons were conducted to assess possible sources of statistical heterogeneity and to detect the influence of the following factors on the association between sleep duration/insomnia and hypertension incidence: length of follow-up, race, sleep duration/insomnia assessment (self-reported or measured (questionnaire producing scores or survey for insomnia), and whether the study included daytime sleep or considered the persistency of insomnia), outcome assessments (measured or not), adjustment for psychological factors (mainly depression) and adjustment for other sleep disorders (such as obstructive sleep apnea) or hypnotics use. The differences between subgroups were assessed by comparing the pooled EE of each subgroup with $\chi^{2}$ and $I^{2}$ analyses and comparing the logarithms of these estimates.

\section{Sensitivity analysis}

Considering a wide variety of study designs among selected studies, a sensitivity analysis was conducted to determine whether the pooled EE was influenced mainly by certain individual studies by omitting one $s$ tudy each time and recalculating the pooled EE of other remaining studies.

A funnel plot was constructed to evaluate the presence of publication bias by plotting the effect measure against the inverse of its s.e.m. The Egger's test was used to estimate the severity of publication bias. When a publication bias was indicated, we recalculated the combined estimate after we imputed the number of 'missing' studies and their effect sizes and s.e.m.s from the asymmetry of the funnel plot using a method known as 'trim and fill' ${ }^{36}$ Statistical analysis was performed with Stata 10.0 and Cochrane Collaboration Review Manager 5.0 software. (The Nordic Cochrane Centre, Copenhagen)

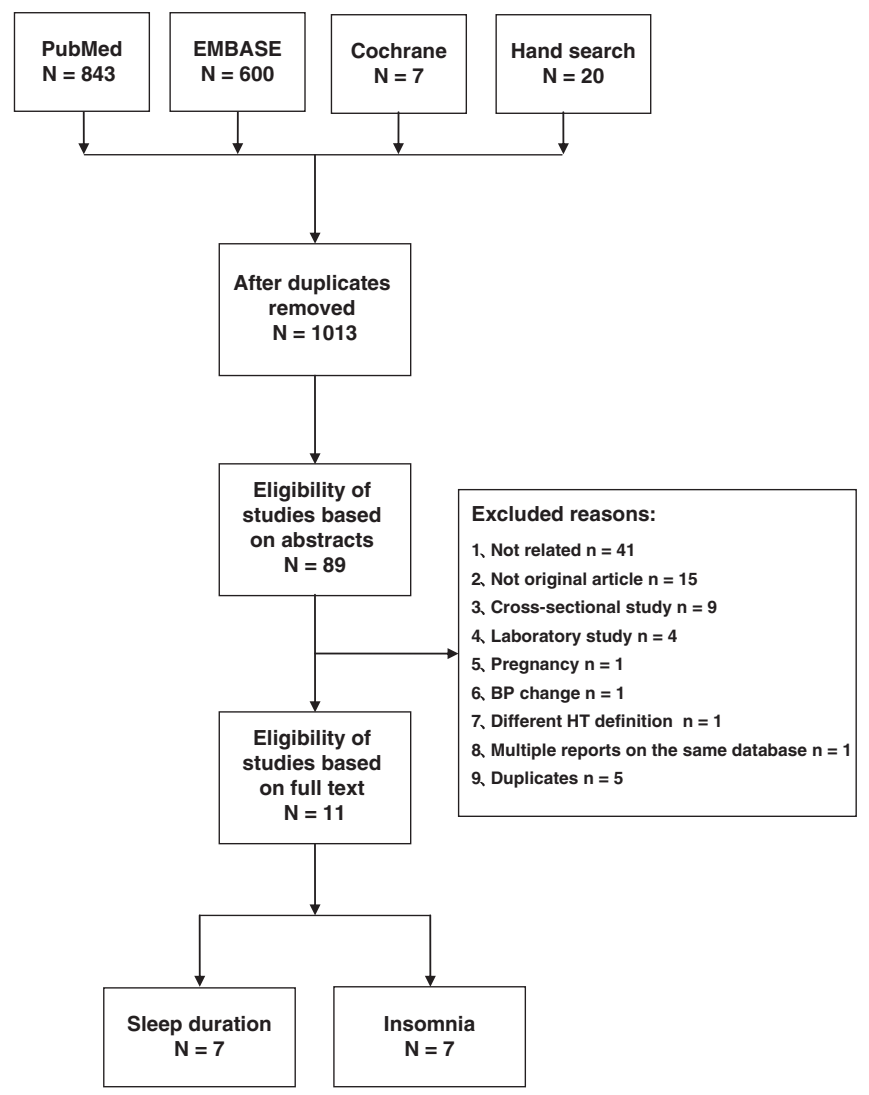

Figure 1 Flowchart of study selection.

\section{RESULTS}

A flow diagram of the study selection process is shown in Figure 1. Eleven studies (17 cohorts) were included, ${ }^{18,22-26,28,30-33}$ and their characteristics are summarized in Table 1. Overall, a total of 58924 participants (22522 for sleep duration and 42636 for insomnia) were included, with a mean follow-up period of 5.5 years for sleep duration and 8.2 years for insomnia, respectively. Four studies were from Europe, ${ }^{30-33}$ five were from the United States, ${ }^{18,22-24,26}$ and two were from Asia. ${ }^{25,28}$ One study ${ }^{25}$ included only men. The remaining 10 studies included both genders; two studies reported results by gender ${ }^{30,33}$ and one study reported results by race-gender categories. $^{23}$ One study measured sleep duration using wrist actigraphy, ${ }^{18}$ and one used polysomnography; ${ }^{24}$ in the remaining studies, sleep duration was assessed using self-reported habitual sleep duration. ${ }^{26,28,30-32}$ One study ${ }^{18}$ assessed sleep continuity/insomnia using wrist actigraphy, four studies assessed insomnia via a survey ${ }^{22-25}$ and two studies assessed insomnia via questionnaires. ${ }^{26,33}$ In two studies, ${ }^{31,32}$ sleep duration was defined as the hours spent sleeping both at night and during the day, whereas other studies defined sleep duration as the hours spent sleeping at night. Three studies ${ }^{24-26}$ took persistency into consideration in their definition of insomnia. The incidence of hypertension was ascertained via self-report in five studies; in the other six studies, hypertension was ascertained with additional BP measurement. In most of the studies, the BP level used to define hypertension was systolic $\mathrm{BP} \geqslant 140 \mathrm{~mm} \mathrm{Hg}$ and/or diastolic $\mathrm{BP} \geqslant 90 \mathrm{~mm} \mathrm{Hg}$; only one study ${ }^{22}$ defined new cases of hypertension as systolic $\mathrm{BP} \geqslant 160 \mathrm{~mm} \mathrm{Hg}$ and/or diastolic $\mathrm{BP} \geqslant 95 \mathrm{~mm} \mathrm{Hg}$. Seven cohorts reported data on the association between hypertension and short sleep, six on long sleep, eight on DFA/difficulty initiating sleep, nine on SCD/difficulty maintaining sleep, six on EMA and nine on combined symptoms of insomnia. The variables that were used in multivariable analyses are shown in Table 2. Overall, the adjusted EEs of almost all the studies were shown after adjustment for age, sex, obesity/body mass index and smoking. Only one study ${ }^{32}$ adjusted for family history of hypertension. Adjustment for exercise/physical activity was made in all but one $\mathrm{e}^{24}$ of the studies on sleep duration and in one study on insomnia. ${ }^{26}$ Five studies adjusted for psychological factors (depression $22,24,30,31$ and stress $^{25}$ ). Seven studies adjusted for other sleep disorders; three of these adjusted for obstructive sleep apnea (sleep-disordered breathing or persistent snoring symptoms), ${ }^{18,24,28}$ three for insomnia or arousal during sleep or daytime sleepiness, ${ }^{26,28,31}$ one for hypnotics use ${ }^{30}$ and one for night work. ${ }^{33}$ Most of the studies assessed sleep disorders using questionnaires or surveys, and only one study assessed sleepdisordered breathing using polysomnography. ${ }^{24}$

All eleven studies were assessed to have moderate quality, with a mean score of 7.5 (range from 5 to 9). The level of agreement between reviewers was $100 \%$.

\section{Short duration of sleep}

Short sleep duration was associated with an increased risk of hypertension incidence (Figure 2a), with no statistical heterogeneity among studies. No evidence of publication bias was found in the funnel plot (Figure 3a) or in Egger's test $(P=0.069)$. All the studies adjusted for other sleep disorders. Longer follow-up duration (relative risk 1.29 (95\% confidence intervals $1.09-1.52)$ ) tended to show a higher incidence of hypertension compared with shorter follow-up duration (1.03 (0.73-1.46)); however, no statistically significant differences were found in terms of subgroup $\left(\chi^{2}=1.09\right.$, degrees of freedom $\left.(\mathrm{df})=1, P=0.30, I^{2}=7.9 \%\right)$ or meta-regression analysis $(P=0.400)$. The subgroup of studies without adjustment for 
Table 1 Overview of studies assessing the association of sleep duration and insomnia with incident hypertension

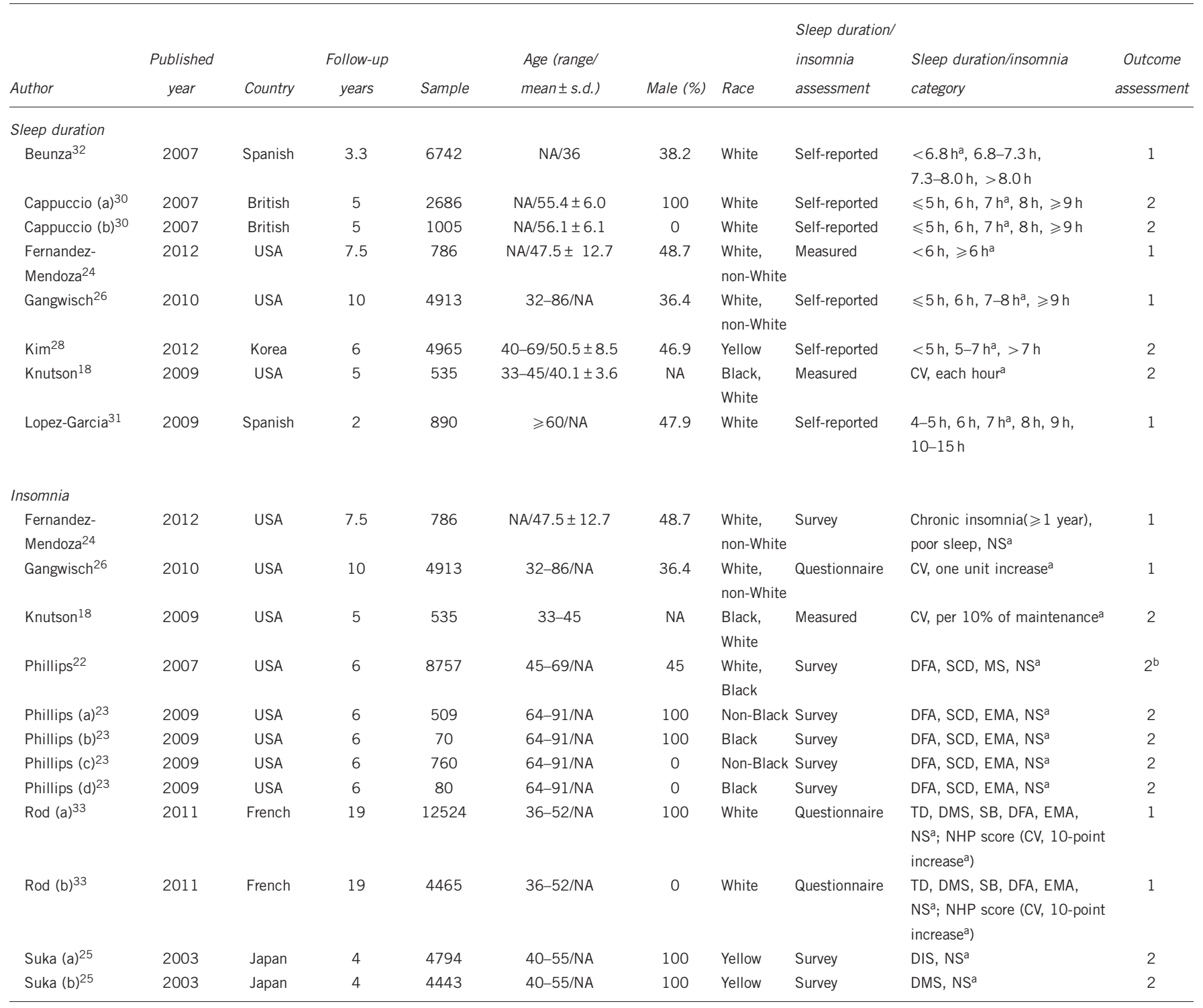

Abbreviations: CV, continuous variables; DBP, diastolic blood pressure; DFA, difficulty falling asleep; DIS, difficulty initiating sleep; DMS, difficulty maintaining sleep; EMA, early-morning awakening; MS, morning sleep; NA, not available; NHP, Nottingham health profile; NS, normal sleep; SB, sleeping bad; SBP, systolic blood pressure; SCD, sleep continuity disturbance; TD, taking drugs for sleep.

(a), (b), (c) and (d) represent different cohorts with separate data reported in a single study.

(a), (b), (c) and (d) represent different cohorts with separate data reported in a single study.
1 represents hypertension incidence that was confirmed by self-reported diagnosis or treatment only; 2 represents hypertension incidence that was confirmed by both self-reported diagnosis or treatment and measurement in the interview at baseline.

a Reference category in each study.

${ }^{\mathrm{b}}$ Measured hypertension is defined as $\mathrm{SBP} \geqslant 160 \mathrm{~mm} \mathrm{Hg}$ and/or DBP $\geqslant 95 \mathrm{~mm} \mathrm{Hg}$, whereas in other articles measured hypertension is defined as $\mathrm{SBP} \geqslant 140 \mathrm{~mm} \mathrm{Hg}$ and/or DBP $\geqslant 90 \mathrm{~mm} \mathrm{Hg}$.

psychological factors had a higher effect (1.29 (1.10-1.52)) compared with the subgroup of studies that adjusted for psychological factors (1.02 (0.72-1.43)); however, the difference was not statistically significant $\left(\chi^{2}=1.63, \mathrm{df}=1, \quad P=0.20, I^{2}=38.5 \%\right)$. Short sleep duration seemed to be more strongly associated with the incidence of hypertension as ascertained via BP measurement (1.24 (1.04-1.49)) than that assessed via self-reported questionnaire (1.11 (0.79-1.57)), but the difference was not significant $\left(\chi^{2}=0.11, \mathrm{df}=1, P=0.75\right.$, $\left.I^{2}=0 \%\right)$. Objective sleep-duration measures seemed to be more strongly associated with the risk of hypertension incidence (1.29 (1.03-1.62)) compared with sleep duration assessed via questionnaires (1.13 (0.90-1.43)), but the differences were not statistically significant $\left(\chi^{2}=0.38, \mathrm{df}=1, P=0.54, I^{2}=0 \%\right)$. After omitting the study in which sleep duration included both daytime and nighttime sleep, ${ }^{31}$ the pooled effect of the remaining studies increased $(1.25$ $(1.09-1.44))$, and differences between these two subgroups were statistically significant $\left(\chi^{2}=4.37, \mathrm{df}=1, P=0.04, I^{2}=77.1 \%\right)$.

\section{Long duration of sleep}

The pooled EEs for long sleep duration were in the direction of decreased risk of hypertension incidence (Figure $2 b$ ), but not significantly. The heterogeneity among studies was mild. There was no evidence of publication bias in the funnel plot (Figure $3 \mathrm{~b}$ ) or in Egger's test $(P=0.146)$. All the studies assessed sleep duration via selfreported questionnaire. Longer follow-up durations (1.07 (0.95-1.21)) tended to show a higher incidence of hypertension compared with 
Table 2 Overview of variables included in multivariable associations of sleep duration and insomnia with incident hypertension

\begin{tabular}{|c|c|c|c|c|c|}
\hline Author & \multicolumn{5}{|c|}{ Adjustment variables } \\
\hline Cappuccio 30 & A & Sm, Al, Ex & $O, D, C V D$ drugs & $\begin{array}{l}\text { Employment, SF-36(men- } \\
\text { tal, physical), depression } \\
\text { cases }\end{array}$ & Hypnotics use \\
\hline Gangwisch ${ }^{26}$ & $A, S, R, E$ & Sm, Al, Ex & $0, D$ & & Sleep duration, insomnia \\
\hline $\mathrm{Kim}^{28}$ & $A, S, E$ & Sm, Al, Ex & $0, B$ & Area, income, job & ESS, snoring \\
\hline Knutson ${ }^{18 a}$ & $A, S, R, E$ & $\begin{array}{l}\text { Sm, Al, Ex (baseline, } \\
\text { 5-year change) }\end{array}$ & $0, D$ & Income & Snoring, daytime sleepiness \\
\hline Lopez- Garcia ${ }^{31}$ & $A, S, E$ & Sm, Al, Ex & $\begin{array}{l}\text { O, number of chronic } \\
\text { diseases }\end{array}$ & $\begin{array}{l}\text { Depression, number of } \\
\text { social ties, anxiolytic intake }\end{array}$ & $\begin{array}{l}\text { Coffee consumption, perceived } \\
\text { health, arousal from sleep }\end{array}$ \\
\hline Suka 25 & A & $\mathrm{Sm}, \mathrm{Al}$ & $O, D$ & Job stress & \\
\hline
\end{tabular}

Abbreviations: A, age; Al, alcohol; AP, angina pectoris; B, baseline blood pressure; C, cholesterol; $\mathrm{CB}$, chronic bronchitis; CHD, coronary heart disease; CVD, cardiovascular disease; D, diabetes; E, education; ESS, Epworth sleepiness scale; Ex, exercise/physical activity; FEV1, forced expiratory volume in one second; $\mathrm{H}$, family history of hypertension; M, marital status; MI, myocardial infarction; O, obesity/body mass index; R, race; S, sex; SDB, sleep-disordered breathing; SF-36, short form-36 health survey questionnaire; Sm, smoking.

${ }^{a}$ All variables were included in the analysis of association of sleep duration on hypertension, whereas only A, S, R were included in the analysis of association of insomnia on hypertension.

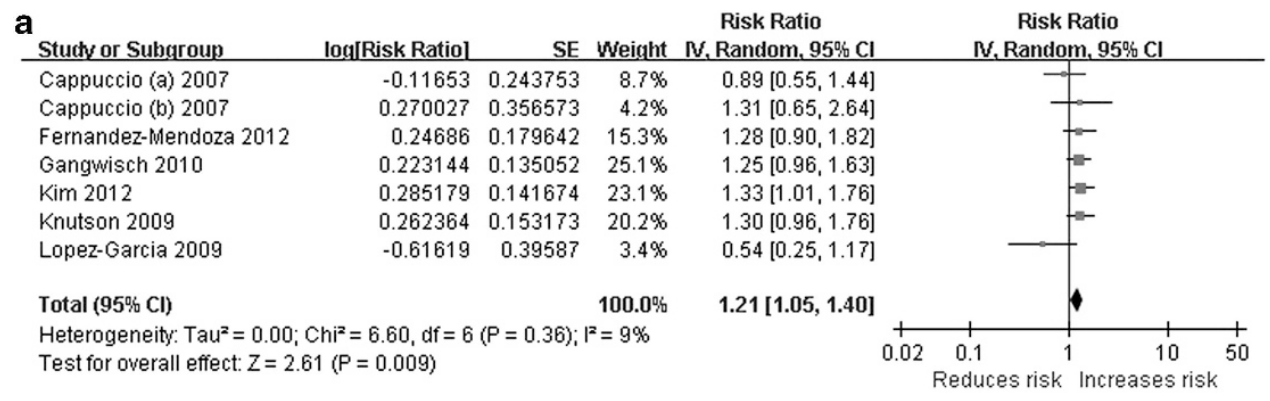

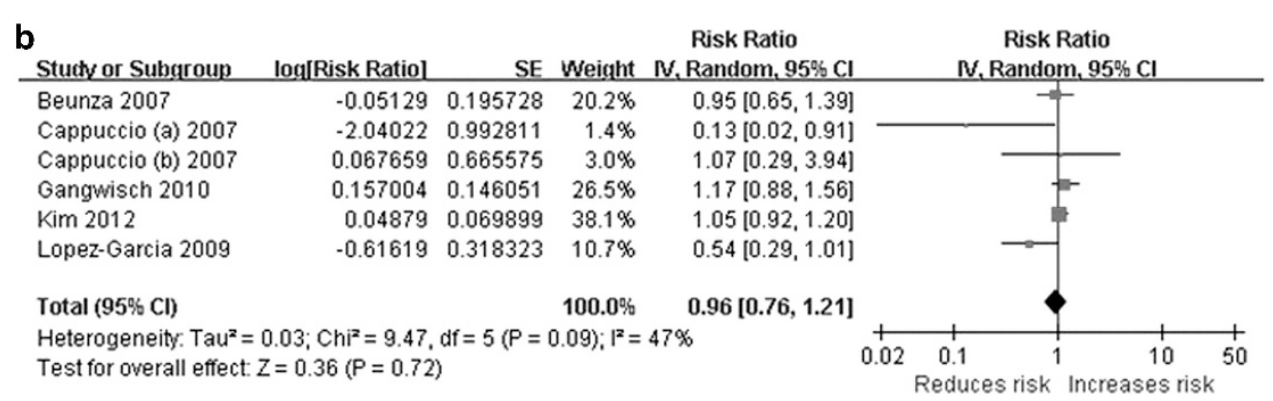

Figure 2 Forest plot of sleep duration and risk of hypertension incidence. (a) Short sleep duration. (b) Long sleep duration. A full-colour version of this figure is available at Hypertension Research online.

shorter follow-up durations $(0.69(0.39-1.22))$; however, no statistically significant differences were found between subgroups $\left(\chi^{2}=3.20\right.$, $\left.\mathrm{df}=1, P=0.07, I^{2}=68.7 \%\right)$ or in the meta-regression analysis $(P=0.242)$. The subgroup of studies without adjustment for psychological factors had a greater effect $(1.06(0.94-1.19))$ than did the subgroup of studies that adjusted for psychological factors $(0.53$ $(0.23-1.21))$, and the difference was statistically significant $\left(\chi^{2}=5.57\right.$, $\left.\mathrm{df}=1, P=0.02, I^{2}=82.0 \%\right)$. The subgroup that included daytime sleep had a smaller effect $(0.76(0.44-1.30))$ than did the subgroup that did not include daytime sleep in the definition of sleep duration (1.06 $(0.82-1.36))$, but the difference was not significant $\left(\chi^{2}=2.24\right.$, $\left.\mathrm{df}=1, P=0.13, I^{2}=55.4 \%\right)$. After omitting the only study that did not adjust for other sleep disorders, ${ }^{32}$ which was also the only study that used the lowest quartile as the reference category for sleep duration, the pooled effect of the remaining studies $(0.93(0.68-1.27))$ was similar with that of all of the included studies. 

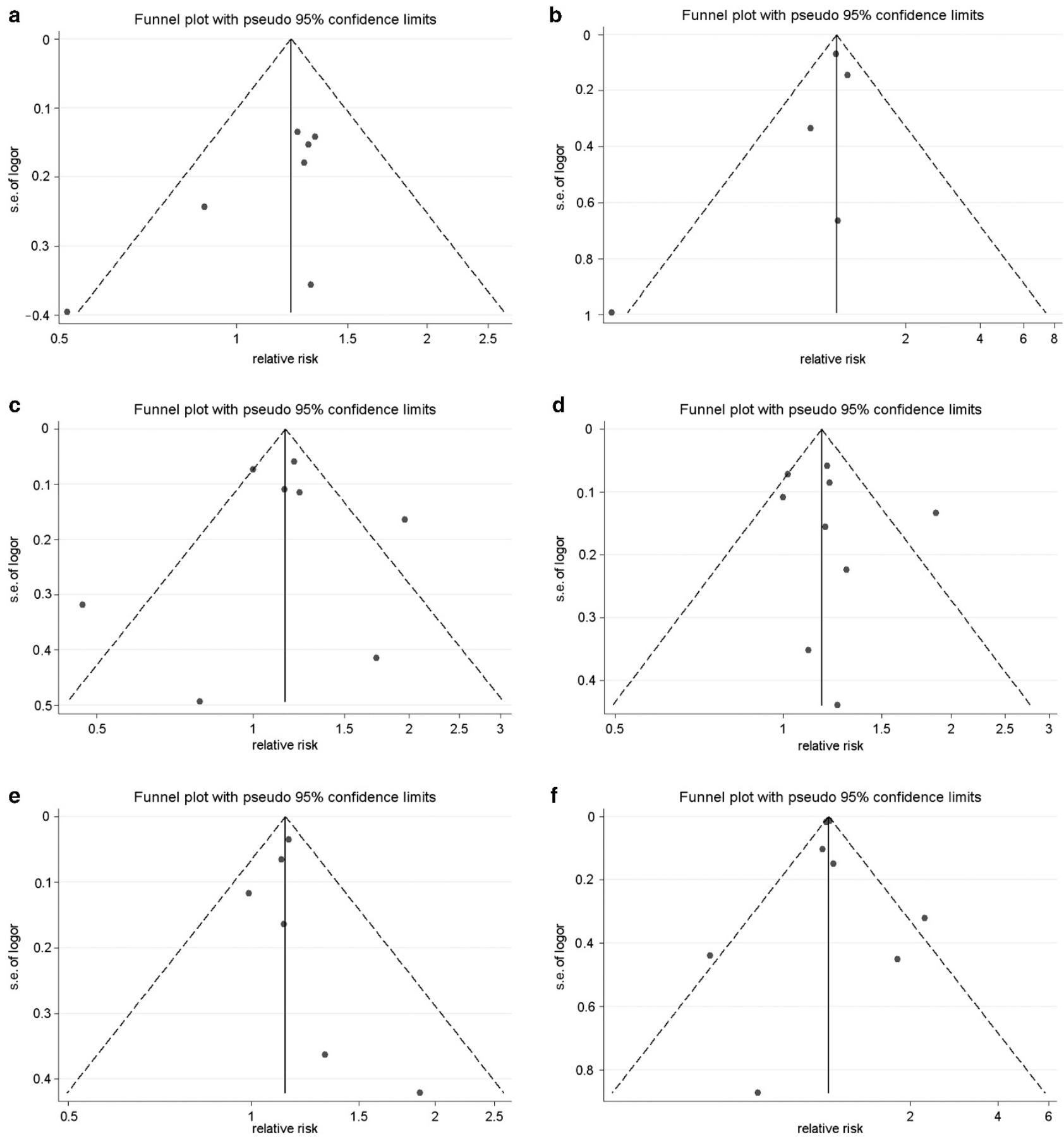

Figure 3 Funnel plot of selected studies. Funnel plot describing the relationship between effect size and s.e. of effect, with possible missing studies imputed. LnRR, natural logarithm of relative risk; vertical line, mean effect size; dashed line, pseudo 95\% confidence limits. (a) Short sleep duration. (b) Long sleep duration. (c) Difficulty falling asleep (DFA). (d) Sleep continuity disturbance (SCD). (e) Early-morning awakening (EMA). (f) Combination of all symptoms of insomnia. A full-colour version of this figure is available at Hypertension Research online.

\section{DFA/difficulty initiating sleep}

The pooled EEs for DFA were in the direction of increased risk of hypertension incidence (Figure 4a), but not significantly so. The heterogeneity among studies was moderate. No evidence of publication bias was found in the funnel plot (Figure 3c) or in Egger's test $(P=0.948)$. Shorter follow-up durations $(1.20(0.91-1.59))$ showed a higher incidence of hypertension, compared with longer follow-up durations (1.05 (0.92-1.19)), and the difference was statistically significant $\left(\chi^{2}=4.51, \mathrm{df}=1, P=0.03, I^{2}=77.8 \%\right)$. However, such significance was not found in meta-regression analysis $(P=0.647)$. Interestingly, the studies that ascertained outcomes using BP measurement, studies that assessed insomnia via survey and studies that did not adjust for other sleep disorders were those that had a shorter follow-up duration. Consequently, the result reported above could be interpreted to mean that DFA was more significantly associated with the incidence of hypertension as ascertained via $\mathrm{BP}$ measurement 


\begin{tabular}{|c|c|c|c|c|}
\hline Stucty or Subqroup & log[Risk Ratio] & SE & Weight & $\begin{array}{c}\text { Risk Ratio } \\
\text { N, Random, } 95 \% \mathrm{Cl}\end{array}$ \\
\hline Phillips (a) 2009 & -0.75502 & 0.318121 & $5.9 \%$ & $0.47[0.25,0.88]$ \\
\hline Phillips (b) 2009 & -0.23572 & 0.493964 & $2.9 \%$ & $0.79[0.30,2.08]$ \\
\hline Phillips (c) 2009 & 0.207014 & 0.115302 & $16.6 \%$ & $1.23[0.98,1.54]$ \\
\hline Phillips (d) 2009 & 0.548121 & 0.414516 & $3.9 \%$ & $1.73[0.77,3.90$ \\
\hline Phillips 2007 & 0.182322 & 0.059389 & $20.8 \%$ & $1.20[1$ \\
\hline Rod (a) 2011 & 0 & 0.073388 & $19.8 \%$ & $1.00[0.87,1.15$ \\
\hline Rod (b) 2011 & 0.139762 & 0.109756 & $17.1 \%$ & $1.15[0.93,1.4$ \\
\hline Suka (a) 2003 & 0.672945 & 0.163927 & $13.0 \%$ & $1.96[1.42,2.7$ \\
\hline \multicolumn{3}{|l|}{ Total $(95 \% \mathrm{Cl})$} & $100.0 \%$ & $1.17[0.98,1.3$ \\
\hline \multicolumn{5}{|c|}{ Heterogeneity: $\operatorname{Tau}^{2}=0.04 ; \mathrm{Chi}^{2}=24.51, \mathrm{df}=7(\mathrm{P}=0.0009) ; \mathrm{I}^{2}=71 \%$} \\
\hline
\end{tabular}

b

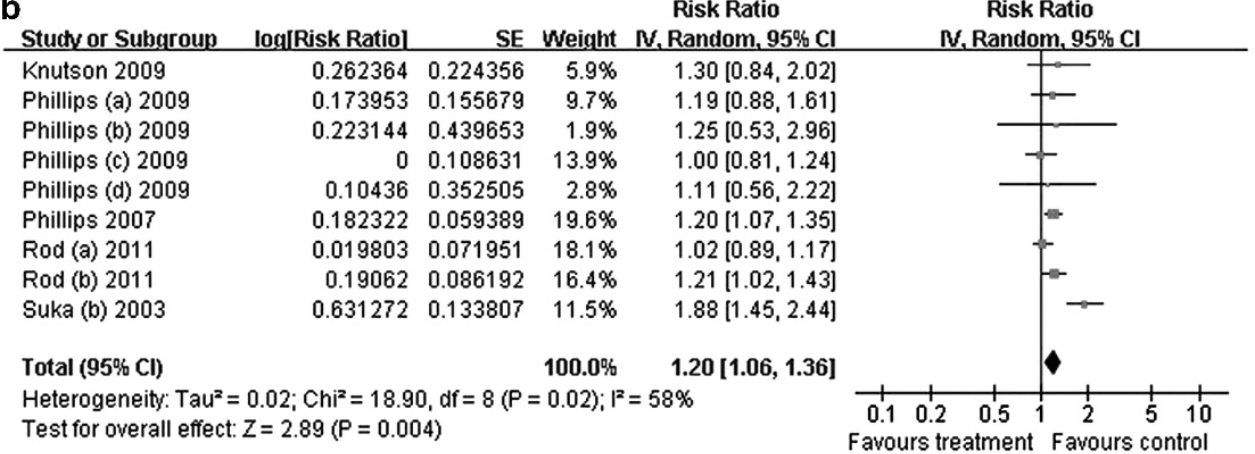

\begin{tabular}{|c|c|c|c|c|c|c|c|}
\hline Stuck or Subgroup & log[Risk Ratio] & SE & Weight & $\begin{array}{l}\text { Risk Ratio } \\
\text { N, Random, } 95 \% \mathrm{Cl}\end{array}$ & $\begin{array}{r}\text { Risk } \\
\text { N. Rando }\end{array}$ & $\begin{array}{l}\text { s Ratio } \\
\mathrm{om}, 95 \% \mathrm{Cl}\end{array}$ & \\
\hline Phillips (a) 2009 & 0.122218 & 0.164066 & $3.2 \%$ & $1.13[0.82,1.56]$ & & & \\
\hline Phillips (b) 2009 & 0.636577 & 0.420813 & $0.5 \%$ & $1.89[0.83,4.31]$ & & & \\
\hline Phillips (c) 2009 & -0.01005 & 0.117058 & $6.4 \%$ & $0.99[0.79,1.25]$ & & & \\
\hline Phillips (d) 2009 & 0.277632 & 0.363274 & $0.7 \%$ & $1.32[0.65,2.69]$ & & & \\
\hline Rod (a) 2011 & 0.139762 & 0.03555 & $69.0 \%$ & $1.15[1.07,1.23]$ & & & \\
\hline Rod (b) 2011 & 0.113329 & 0.065538 & $20.3 \%$ & $1.12[0.98,1.27]$ & & $=$ & \\
\hline Total $(95 \% \mathrm{Cl})$ & & & $100.0 \%$ & $1.14[1.07,1.20]$ & & 1 & \\
\hline \multicolumn{5}{|c|}{$\begin{array}{l}\text { Heterogeneity: } \mathrm{Tau}^{2}=0.00 ; \mathrm{Chi}^{2}=3.18, \mathrm{df}=5(P=0.67) ; \mathrm{I}^{2}=0 \% \\
\text { Test for overall effect: } Z=4.32(P<0.0001)\end{array}$} & $\begin{array}{lll}0.1 & 0.2 & 0.5 \\
& \text { Reduces risk }\end{array}$ & 12 & $\begin{array}{l}510 \\
\text { es risk }\end{array}$ \\
\hline
\end{tabular}

\begin{tabular}{|c|c|c|c|c|c|c|c|}
\hline Stucty or Subqroup & log[Risk Ratio] & SE & Weight & $\begin{array}{c}\text { Risk Ratio } \\
\text { N. Random, } 95 \% \text { Cl }\end{array}$ & $\begin{array}{r}\text { Risk } \\
\text { N. Rando }\end{array}$ & $\begin{array}{l}\text { Ratio } \\
\mathrm{m}, 95 \% \mathrm{Cl}\end{array}$ & \\
\hline Fernandez-Mendoza 2012 & 0.806476 & 0.321109 & $0.3 \%$ & $2.24[1.19,4.20]$ & & & \\
\hline Gangwisch 2010 & 0.029559 & 0.01726 & $29.0 \%$ & $1.03[1.00,1.07]$ & & & \\
\hline Phillips (a) 2009 & -0.8916 & 0.438945 & $0.1 \%$ & $0.41[0.17,0.97]$ & & & \\
\hline Phillips (b) 2009 & -0.51083 & 0.873007 & $0.0 \%$ & $0.60[0.11,3.32]$ & & & \\
\hline Phillips (c) 2009 & 0.086178 & 0.150295 & $1.1 \%$ & $1.09[0.81,1.46]$ & & & \\
\hline Phillips (d) 2009 & 0.593327 & 0.451349 & $0.1 \%$ & $1.81[0.75,4.38]$ & & & \\
\hline Phillips 2007 & 0 & 0.103435 & $2.3 \%$ & $1.00[0.82,1.22]$ & & t & \\
\hline Rod (a) 2011 & 0.04879 & 0.012208 & $34.7 \%$ & $1.05[1.03,1.08]$ & & 息 & \\
\hline Rod (b) 2011 & 0.058269 & 0.014444 & $32.2 \%$ & $1.06[1.03,1.09]$ & & 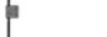 & \\
\hline Total $(95 \% \mathrm{Cl})$ & & & $100.0 \%$ & $1.05[1.01,1.08]$ & & & \\
\hline \multicolumn{5}{|c|}{$\begin{array}{l}\text { Heterogeneity. } \text { Tau }^{2}=0.00 ; \mathrm{Chi}^{2}=13.96, \mathrm{df}=8(P=0.08) ; \mathrm{I}^{2}=43 \% \\
\text { Test for overall effect: } Z=2.87(P=0.004)\end{array}$} & $\begin{array}{rrr}0.1 & 0.2 & 0.5 \\
& \text { Reduces risk }\end{array}$ & 12 & $\begin{array}{l}510 \\
5 \text { risk }\end{array}$ \\
\hline
\end{tabular}

Figure 4 Forest plot of symptoms of insomnia and risk of hypertension incidence. (a) DFA (b) SCD. (c) EMA. (d) Combination of all symptoms of insomnia. A full-colour version of this figure is available at Hypertension Research online.

than with BP assessed via self-reported questionnaires, or that DFA ascertained via survey showed a greater effect on hypertension incidence than did DFA ascertained via quantitative questionnaire, or that studies that adjusted for other sleep disorders had a smaller effect than the studies that did not adjust for other sleep disorders. The subgroup of studies that did not adjust for psychological factors had a smaller effect $(1.05(0.86-1.28))$ than did the subgroup of studies that adjusted for psychological factors $(1.50(0.93-2.42))$, and the difference was statistically significant $\left(\chi^{2}=5.46, \mathrm{df}=1, P=0.02\right.$,
$I^{2}=81.7 \%$ ). Subgroup analysis stratified by race (African, Asian and multiracial) found statistically significant differences between subgroups $\left(\chi^{2}=11.13, \mathrm{df}=2, P=0.004, I^{2}=82 \%\right)$. When the subgroup stratified as Asian only and other races, the differences became more significant $\left(\chi^{2}=11.00, \mathrm{df}=1, P=0.0009, I^{2}=90.9 \%\right)$. After omitting the only study that included the Asian, ${ }^{25}$ which was also the study in which persistency of insomnia was included in the insomnia definition, the pooled effect was attenuated (1.10 (0.95-1.27)). No significant differences were found between subgroups of studies 
that included African and multiracial subjects $\left(\chi^{2}=0.12, \mathrm{df}=1\right.$, $\left.P=0.73, I^{2}=0 \%\right)$.

\section{SCD/difficulty maintaining sleep}

SCD was associated with an increased risk of hypertension incidence (Figure $4 \mathrm{~b}$ ), with no evidence of publication bias in the funnel plot (Figure $3 \mathrm{~d})$ or in Egger's test $(P=0.549)$. The heterogeneity among studies was moderate. The subgroup with a shorter follow-up duration (1.26 (1.06-1.50)) showed a greater effect on the risk of hypertension incidence compared with the subgroup with a longer follow-up duration $(1.10(0.93-1.30))$, but the difference was not statistically significant $\left(\chi^{2}=2.54, \mathrm{df}=1, P=0.11, I^{2}=60.6 \%\right)$. No significant differences in follow-up duration were found in the metaregression analysis $(P=0.330)$. Interestingly, the studies in which the outcome was ascertained using BP measurement and the studies that did not adjust for other sleep disorders were those with shorter follow-up durations. Consequently, the above result, although not significant, could also be interpreted to mean that SCD was more strongly associated with the incidence of hypertension ascertained by the BP measurement than with that assessed via self-reported questionnaire, or that studies that adjusted for other sleep disorders showed a smaller effect than studies that did not adjust for other sleep disorders. None of the studies adjusted for both psychological factors and other sleep disorders, and the two studies adjusted for neither. ${ }^{18,23}$ The subgroup of studies that adjusted for psychological factors had greater effect (1.48 (0.95-2.29)) than did the subgroup of studies that did not adjust for psychological factors (1.09 (1.00$1.20)$ ), with statistically significant differences $\left(\chi^{2}=5.52, \mathrm{df}=1\right.$, $\left.P=0.02, \quad I^{2}=81.9 \%\right)$. After omitting the only study that used objective sleep measurement, ${ }^{18}$ the pooled effect of the remaining studies increased slightly $(1.20(1.05-1.36))$. No significant differences were found between the subgroup that used objective sleep measurement and the subgroup that used self-reported symptoms $\left(\chi^{2}=0.22, \mathrm{df}=1, P=0.64, I^{2}=0 \%\right)$. The effect in subgroup of studies with insomnia assessed by survey (1.25 (1.03-1.52)) was higher than that in the subgroup of studies that assessed insomnia via questionnaire $(1.10(0.93-1.30))$ or wrist actigraphy $(0.77$ $(0.50-1.19))$, but the difference was not significant $\left(\chi^{2}=5.80\right.$, $\left.\mathrm{df}=2, \quad P=0.06, I^{2}=65.5 \%\right)$. A subgroup analysis stratified by race (African, Asian and multiracial) found statistically significant differences between subgroups $\left(\chi^{2}=13.41, \mathrm{df}=2, P=0.0001, I^{2}=\right.$ $85.1 \%)$. When the subgroups were stratified as Asian only and other races, the differences remained significant $\left(\chi^{2}=13.40, \mathrm{df}=1\right.$, $\left.P=0.0003, I^{2}=92.5 \%\right)$. After omitting the only study that included the Asian, ${ }^{25}$ which was also the study in which persistency of insomnia was included in the insomnia definition, the pooled effect slightly attenuated (1.13 (1.05-1.21)), but remained positively associated. No significant differences were found between subgroups of studies that included African and multiracial subjects $\left(\chi^{2}=0.01\right.$, $\left.\mathrm{df}=1, P=0.92, I^{2}=0 \%\right)$. The pooled EEs were positive despite the omission of any one selected study, indicating that the overall result was not influenced significantly by any individual study.

\section{Early-morning awakening}

EMA was associated with an increased risk of hypertension incidence (Figure 4c), with no evidence of publication bias in the funnel plot (Figure $3 \mathrm{e})$ or in Egger's test $(P=0.720)$ and no heterogeneity among studies. Because the included cohorts originated from only two studies, the subgroup analysis was conducted only for race (African and multiracial), and no statistically significant differences were found between subgroups $\left(\chi^{2}=1.23, \mathrm{df}=1, P=0.27, I^{2}=18.7 \%\right)$. There were no statistically significant differences in meta-regression on follow-up duration $(P=0.720)$. The pooled EEs became borderline (1.11(1.00-1.23)) only when the cohort of Rod (a) was omitted in the sensitivity analysis.

\section{Combination of all symptoms of insomnia}

The combination of all insomnia symptoms increased the risk of hypertension incidence (Figure 4d), with no evidence of publication bias in the funnel plot (Figure 3f) or in Egger's test $(P=0.911)$. The heterogeneity among studies was mild. There were no statistically significant differences in the subgroup analysis stratified by length of follow-up duration $\left(\chi^{2}=0.02, \mathrm{df}=1, P=0.89, I^{2}=0 \%\right)$ or in the meta-regression on follow-up duration $(P=0.434)$. The subgroup analysis stratified by race (African, white only and multiracial) found no statistically significant differences $\left(\chi^{2}=1.97, \mathrm{df}=2, P=0.37\right.$, $\left.I^{2}=0 \%\right)$. There were also no significant differences between subgroups stratified by insomnia assessment $\left(\chi^{2}=0.02, \mathrm{df}=1, P=0.89\right.$, $\left.I^{2}=0 \%\right)$, outcome assessment $\left(\chi^{2}=0.22, \mathrm{df}=1, P=0.64, I^{2}=0 \%\right)$, adjustment for psychological factors $\left(\chi^{2}=0.08, \mathrm{df}=1, P=0.77\right.$, $\left.I^{2}=0 \%\right)$, adjustment for other sleep disorders $\left(\chi^{2}=0.22, \mathrm{df}=1\right.$, $\left.P=0.64, I^{2}=0 \%\right)$ or the inclusion of persistency of insomnia $\left(\chi^{2}=1.09, \mathrm{df}=1, P=0.30, I^{2}=7.9 \%\right)$.

No statistically significant differences were found in the metaregression analysis on baseline male percentages and sample size in any sleep-duration and insomnia group. No significant differences were found in the meta-regression analysis on mean age at baseline in any sleep-duration group. We were unable to stratify studies on insomnia by age groups because of the inconsistent reporting of age in the original studies.

\section{DISCUSSION}

This is the first meta-analysis focusing on the contribution of both habitual sleep duration and insomnia symptoms to the risk of hypertension incidence. In general, the present meta-analysis indicates that short sleep duration, SCD, EMA and combined symptoms of insomnia are associated with an increased risk of hypertension incidence, and the association was statistically significant. Less evidence exists to support conclusions regarding the associations between long sleep duration or DFA and hypertension incidence, although the tendency for long sleep duration is in the direction of decreased risk of incidence of hypertension, whereas that for DFA is in the direction of increased risk. The present study is robust because of the prospective study design, which minimized the selection and recall bias. ${ }^{37}$ The absence of severe statistical heterogeneity among studies and no evidence of publication bias further strengthen our results. No definite evidence was found in either subgroup or meta-regression analyses to prove the influence of follow-up duration on the association between sleep duration/insomnia symptoms and hypertension incidence. The effects were comparable in men and women and did not depend on either the objective/subjective assessment of exposure and outcome (with the exception of DFA) or the differences in categorization of short or long sleep duration. However, the inclusion of daytime sleep in the definition of sleep duration or persistency in the definition of insomnia showed some inconsistent influence on the association between sleep duration/insomnia and hypertension. A large number of potential confounding factors (particularly age, sex, obesity/body mass index and smoking) were considered in the primary analyses. There was no significant influence of race on the association between sleep duration and hypertension incidence; however, the effect of insomnia symptoms on hypertension incidence was probably affected by racial differences. When the Asian 
study was added, the subgroup analysis became significantly different in terms of DFA and SCD. When that study was omitted, no statistically significant differences were found between subgroups stratified by race. However, the Asian study was also the study that considered persistency in the definition of insomnia, so it was difficult to conclude whether the effect was influenced by race or by differences in insomnia definitions. Another factor influencing the effect of the present study is the adjustment of psychological factors and other sleep disorders. For DFA and SCD, the effect in the subgroup of studies that adjusted for psychological factors was higher than that of the subgroup of studies that did not adjust for psychological factors; this result could mean that the effect of DFA and SCD on hypertension incidence can be attributed partly to the effect of psychological factors, or that the existence of psychological factors might underestimate the effect of insomnia symptoms on hypertension incidence. The adjustment of other sleep disorders attenuated the effect of DFA (significantly) and SCD (nonsignificantly) on hypertension incidence, which meant that the association between insomnia symptoms and hypertension incidence could be partly explained by other sleep disorders, such as untreated sleep apnea with insomnia, or that the variance explained by insomnia overlaps, even if it is genuinely caused by the insomnia. For sleep-duration studies, the effect in the subgroup with adjustment of psychological factors was smaller than in the subgroup without adjustment, which meant that if all studies adjusted for psychological factors in sleep-duration studies, the effect might be smaller than the present result. The association between sleep duration and incidence of hypertension could be partly explained by the existence of psychological factors.

\section{Study limitations}

It is important to notice that the quality and quantity of selected studies might attenuate the strength of the result to some degree.

First, a meta-analysis of observational data cannot directly control for confounding. ${ }^{14}$ In the present meta-analysis, adjusted estimates from multivariate models were included, and subgroup analysis was conducted (with subgroups stratified by the adjustment for psychological factors and other sleep disorders) to assess the potential influence of confounding. However, residual confounding and bias remain a possibility. Furthermore, as discussed above, the inadequate adjustment of confounding may affect the strength of our results. Second, the inconsistent influence of daytime sleep (in the definition of sleep duration) or persistency (in the definition of insomnia) on the association between sleep and hypertension may be the result of inconsistent sleep assessment methods among studies, such as various combinations of insomnia symptoms, different levels of insomnia persistency and different interpretations of sleep time. The relatively small number of studies included in the present metaanalysis restricted the application of subgroup analysis and might weaken the strength of the results to some extent; however, little or no heterogeneity and no evidence of publication bias demonstrate that our results are robust. Third, all the studies assessed sleep duration and insomnia at baseline only, ignoring possible fluctuations or changes with time. In addition, most of selected studies assessed sleep duration and insomnia using self-reported questionnaires. These methods have limitations in that they often may not allow differentiation between time asleep and time in bed or the estimation of the number and duration of naps when assessing sleep duration (unless these aspects are explicitly addressed in additional questions). ${ }^{14}$ A recent analysis comparing sleep durations estimated from wrist actigraphy to self-reported sleep in a population-based sample of 669 middle-aged adults indicated only a moderate correlation between these measures $(r=0.47)$, but these results were biased by systematic over-reporting. ${ }^{38}$ However, sleep studies using objective measures of sleep are not practical and often not feasible in large, prospective population studies. There were also studies that found that self-reported assessments of sleep were valid measures compared with quantitative sleep assessments via actigraphy. ${ }^{39,40}$ In our meta-analysis, no difference was found in the effects of including or omitting the study that assessed sleep duration via actigraphy. Fourth, none of the selected studies used 24-h ambulatory BP monitoring, and none assessed the association between sleep and nocturnal hypertension. Furthermore, the results can only be representative of the studies that were included. Most of the studies were conducted in Europe, the United States, Korea and Japan; therefore, these studies cannot represent other world populations, such as those from India, Australia or Africa. We were unable to stratify studies on insomnia by age groups because of the inconsistent reporting of age in the original studies.

\section{Potential mechanisms}

BP gradually falls with the onset of sleep and then remains low until awakening, when it promptly rises. ${ }^{41}$ Current studies employ laboratory-controlled sleep deprivation to examine outcomes associated with sleep loss, which may generalize to short sleep. ${ }^{8}$ Laboratory studies of short-term sleep deprivation have suggested potential mechanisms for a causal link between sleep loss and hypertension. Some sleep deprivation studies have found that BP and sympathetic nervous system activity increased significantly after sleep restriction, ${ }^{15,16,42}$ and intervention study with melatonin (a hormone secreted at night that affects many physiological functions, including sleep) has been effective at reducing $\mathrm{BP}^{17}$ Other contributing mechanisms may include overactivity of the renin-angiotensin-aldosterone system, proinflammatory responses, endothelial dysfunction, renal impairment ${ }^{43,44}$ and alteration of the circadian rhythm. ${ }^{45}$ Sleep restriction has also been shown to compromise insulin sensitivity ${ }^{46}$ and to increase appetite by decreasing leptin and increasing ghrelin, ${ }^{47}$ which could eventually induce type 2 diabetes and obesity, both common risk factors for hypertension. By contrast, it has been suggested that physical inactivity, alcohol consumption and depression, which are more common among people with long sleep durations, may raise BP. ${ }^{19}$ Epidemiological studies have also shown a U-shaped association between sleep duration and all-cause mortality risk, and long sleep duration may be more detrimental in terms of mortality than short sleep. ${ }^{48}$ Proposed mechanisms for mortality associated with long sleep include the following: (1) sleep fragmentation, (2) fatigue, (3) immune function, (4) photoperiodic abnormalities, (5) lack of challenge, (6) depression and (7) an underlying disease process, such as (a) sleep apnea, (b) heart disease or (c) failing health. The generally strongly supported mechanisms in the current literature include depression, photoperiodic abnormalities and health risks. Moreover, long sleepers demonstrate some differences in physiology (such as rhythms of melatonin, temperature, timing and activity) and in electroencephalographic measurements compared with short sleepers; ${ }^{49}$ these differences indicate that the mechanism underlying the association between long sleep duration and mortality may differ from the mechanisms underlying the association between short sleep and mortality. The meta-analysis also shows a U-shaped association between sleep duration and diabetes risk; the effect of long sleep duration is greater than that of short sleep duration. ${ }^{14}$ However, such an association was not found in the present meta-analysis of prospective studies on the association between sleep duration and 
hypertension; in other words, short sleep duration, but not long sleep duration, was associated with increased hypertension incidence, whereas long sleep duration showed a trend in the direction of decreased hypertension risk. This finding indicates that the increased mortality associated with long sleep duration is probably mediated by other mechanisms, but not by increasing hypertension risk. Furthermore, the present meta-analysis found that the subgroup of studies that did not adjust for psychological factors had a significantly greater effect than the subgroup of studies that did adjust for psychological factors, indicating that increased hypertension risk in long sleepers may in fact be mediated by psychological factors, such as depression. More studies are needed to further assess the association between long sleep duration and hypertension and the mechanisms through which long sleep duration affect hypertension incidence.

It is now known that patients with primary insomnia have increased high-frequency electroencephalogram activation, abnormal hormone secretion, increased whole-body and brain metabolic activation and elevated heart rates and sympathetic nervous system activation throughout day and night. ${ }^{50}$ If physiological activation is chronic in insomnia patients, then these patients should be at increased risk for abnormalities associated with elevated sympathetic nervous system or hypothalamic-pituitary-adrenal activity, such as hypertension and other cardiovascular disorders. ${ }^{51}$ In addition, some studies $^{20,24}$ found that insomnia and short sleep duration had a synergistic effect on hypertension and that insomnia chronicity (duration) had a strong effect on the association between insomnia symptoms and hypertension. Our meta-analysis also found an effect of insomnia persistency (duration) in the association between DFA/ SCD and hypertension incidence. However, no such insomnia persistency effect was found in the association between combined symptoms of insomnia and hypertension incidence. In addition, not all of the insomnia symptoms significantly increased the risk of hypertension incidence in our meta-analysis, indicating that different components of insomnia could have different outcomes through different mechanisms. These inconsistencies in hypertension incidence associated with insomnia also indicate that the mechanisms underlying the hypertension incidence associated with insomnia differ from those associated with short sleep duration.

\section{CONCLUSIONS}

Our meta-analysis supports that short sleep duration, SCD, EMA and combined symptoms of insomnia are associated with an increased risk of hypertension. Less evidence exists to support conclusions about the associations between long sleep duration or DFA and hypertension incidence. The association between sleep duration/ insomnia (at least certain symptoms of insomnia) and hypertension may vary among different races, and the Asian population in particular might differ considerably from other populations. It is important to consider sleep duration and insomnia in hypertension prevention and treatment. More laboratory studies are needed to further detect potential biological mechanisms. Prospective observational studies with objective measures of sleep are needed. Future studies should also consider adequate follow-up duration, nocturnal hypertension, uniform definitions of insomnia, multipoint assessments of exposure, adequate adjustment for psychological factors and other sleep disorders and possible variations by age, gender and race.

\section{ACKNOWLEDGEMENTS}

The study was supported by the Key Project in the National Science and Technology Pillar Program during the 12th 5-Year Plan Period (2011BAI11B04).
1 Stenberg D. Neuroanatomy and neurochemistry of sleep. Cell Mol Life Sci 2007; 64 1187-1204.

2 Rechtschaffen A, Bergmann BM, Everson CA, Kushida CA, Gilliland MA. Sleep deprivation in the rat: $X$. Integration and discussion of the findings. Sleep 1989; 12: 68-87.

3 Mitchell HA, Weinshenker D. Good night and good luck: norepinephrine in sleep pharmacology. Biochem Pharmacol 2010; 79: 801-809.

4 Tasali E, Leproult R, Spiegel K. Reduced sleep duration or quality: relationships with insulin resistance and type 2 diabetes. Prog Cardiovasc Dis 2009; 51: 381-391.

5 Riemann D, Spiegelhalder K, Feige B, Voderholzer U, Berger M, Perlis M, Nissen C. The hyperarousal model of insomnia: a review of the concept and its evidence. Sleep Med Rev 2010; 14: 19-31.

6 Ebben MR, Spielman AJ. Non-pharmacological treatments for insomnia. J Behav Med 2009; 32: 244-254.

7 Knutson KL. Sleep duration and cardiometabolic risk: a review of the epidemiologic evidence. Best Pract Res Clin Endocrinol Metab 2010; 24: 731-743.

8 Grandner MA, Patel NP, Gehrman PR, Perlis ML, Pack AI. Problems associated with short sleep: bridging the gap between laboratory and epidemiological studies. Sleep Med Rev 2010; 14: 239-247.

9 Akerstedt T, Nilsson PM. Sleep as restitution: an introduction. J Intern Med 2003, 254: 6-12.

10 Gangwisch JE, Malaspina D, Boden-Albala B, Heymsfield SB. Inadequate sleep as a risk factor for obesity: analyses of the nhanes i. Sleep 2005; 28: 1289-1296.

11 Gangwisch JE, Heymsfield SB, Boden-Albala B, Buijs RM, Kreier F, Pickering TG, Rundle AG, Zammit GK, Malaspina D. Short sleep duration as a risk factor for hypertension: analyses of the first national health and nutrition examination survey. Hypertension 2006; 47: 833-839.

12 Kripke DF, Garfinkel L, Wingard DL, Klauber MR, Marler MR. Mortality associated with sleep duration and insomnia. Arch Gen Psychiatry 2002; 59: 131-136.

13 Sutton DA, Moldofsky H, Badley EM. Insomnia and health problems in Canadians. Sleep 2001; 24: 665-670.

14 Cappuccio FP, D'Elia L, Strazzullo P, Miller MA. Quantity and quality of sleep and incidence of type 2 diabetes: a systematic review and meta-analysis. Diabetes Care 2010; 33: 414-420.

15 Tochikubo O, Ikeda A, Miyajima E, Ishii M. Effects of insufficient sleep on blood pressure monitored by a new multibiomedical recorder. Hypertension 1996; 27: 1318-1324.

16 Lusardi P, Zoppi A, Preti P, Pesce RM, Piazza E, Fogari R. Effects of insufficient sleep on blood pressure in hypertensive patients: a 24-h study. Am J Hypertens 1999; 12 63-68.

17 Scheer FA, Van Montfrans GA, van Someren EJ, Mairuhu G, Buijs RM. Daily nighttime melatonin reduces blood pressure in male patients with essential hypertension. Hypertension 2004; 43: 192-197.

18 Knutson KL, Van Cauter E, Rathouz PJ, Yan LL, Hulley SB, Liu K, Lauderdale DS. Association between sleep and blood pressure in midlife: the cardia sleep study. Arch Intern Med 2009; 169: 1055-1061.

19 Gottlieb DJ, Redline S, Nieto FJ, Baldwin CM, Newman AB, Resnick HE, Punjabi NM. Association of usual sleep duration with hypertension: the sleep heart health study. Sleep 2006; 29: 1009-1014.

20 Vgontzas AN, Liao D, Bixler EO, Chrousos GP, Vela-Bueno A. Insomnia with objective short sleep duration is associated with a high risk for hypertension. Sleep 2009; 32 : 491-497.

21 Friedman O, Shukla Y, Logan AG. Relationship between self-reported sleep duration and changes in circadian blood pressure. Am J Hypertens 2009; 22: 1205-1211.

22 Phillips B, Mannino DM. Do insomnia complaints cause hypertension or cardiovascular disease? J Clin Sleep Med 2007; 3: 489-494.

23 Phillips B, Buzkova P, Enright P. Insomnia did not predict incident hypertension in older adults in the cardiovascular health study. Sleep 2009; 32: 65-72.

24 Fernandez-Mendoza J, Vgontzas AN, Liao D, Shaffer ML, Vela-Bueno A, Basta M, Bixler EO. Insomnia with objective short sleep duration and incident hypertension: the penn state cohort. Hypertension 2012; 60: 929-935.

25 Suka M, Yoshida K, Sugimori H. Persistent insomnia is a predictor of hypertension in Japanese male workers. J Occup Health 2003; 45: 344-350.

26 Gangwisch JE, Malaspina D, Posner K, Babiss LA, Heymsfield SB, Turner JB, Zammit GK Pickering TG. Insomnia and sleep duration as mediators of the relationship between depression and hypertension incidence. Am J Hypertens 2010; 23: 62-69.

27 Wang Q, Xi B, Liu M, Zhang Y, Fu M. Short sleep duration is associated with hypertension risk among adults: A systematic review and meta-analysis. Hypertens Res 2012; 35: 1012-1018.

28 Kim SJ, Lee SK, Kim SH, Yun CH, Kim JH, Thomas RJ, Shin C. Genetic association of short sleep duration with hypertension incidence-a 6-year follow-up in the Korean genome and epidemiology study. Circ J 2012; 76: 907-913.

29 Liberati A, Altman DG, Tetzlaff J, Mulrow C, Gotzsche PC, loannidis JP, Clarke M, Devereaux PJ, Kleijnen J, Moher D. The prisma statement for reporting systematic reviews and meta-analyses of studies that evaluate health care interventions: explanation and elaboration. Ann Intern Med 2009; 151: W65-W94.

30 Cappuccio FP, Stranges S, Kandala NB, Miller MA, Taggart FM, Kumari M, Ferrie JE, Shipley MJ, Brunner EJ, Marmot MG. Gender-specific associations of short sleep duration with prevalent and incident hypertension: The whitehall ii study. Hypertension 2007; 50: 693-700.

31 Lopez-Garcia E, Faubel R, Guallar-Castillon P, Leon-Munoz L, Banegas JR, RodriguezArtalejo F. Self-reported sleep duration and hypertension in older spanish adults. J Am Geriatr Soc 2009; 57: 663-668. 
32 Beunza JJ, Martinez-Gonzalez MA, Ebrahim S, Bes-Rastrollo M, Nunez J, Martinez JA, Alonso A. Sedentary behaviors and the risk of incident hypertension: the sun cohort. Am J Hypertens 2007; 20: 1156-1162.

33 Rod NH, Vahtera J, Westerlund H, Kivimaki M, Zins M, Goldberg M, Lange T. Sleep disturbances and cause-specific mortality: results from the gazel cohort study. Am J Epidemiol 2011; 173: 300-309.

34 Wells GA, Shea B, O'Connell D, Peterson J, Welch V, Losos M, Tugwell P. The newcastle-ottawa scale (nos) for assessing the quality of nonrandomised studies in meta-analyses. Available at: http://www.ohri.ca/programs/clinical_epidemiology/oxford. htm. (accessed 7 September, 2010). 2008.

35 Thompson SG, Higgins JP. How should meta-regression analyses be undertaken and interpreted? Stat Med 2002; 21: 1559-1573.

36 Sutton AJ, Duval SJ, Tweedie RL, Abrams KR, Jones DR. Empirical assessment of effect of publication bias on meta-analyses. BMJ 2000; 320: 1574-1577.

$37 \mathrm{He}$ FJ, Nowson CA, MacGregor GA. Fruit and vegetable consumption and stroke: metaanalysis of cohort studies. Lancet 2006; 367: 320-326.

38 Lauderdale DS, Knutson KL, Yan LL, Liu K, Rathouz PJ. Self-reported and measured sleep duration: how similar are they? Epidemiology 2008; 19: 838-845.

39 Hauri PJ, Wisbey J. Wrist actigraphy in insomnia. Sleep 1992; 15: 293-301.

40 Lockley SW, Skene DJ, Arendt J. Comparison between subjective and actigraphic measurement of sleep and sleep rhythms. J Sleep Res 1999; 8: 175-183.

41 Kario K, Schwartz JE, Pickering TG. Changes of nocturnal blood pressure dipping status in hypertensives by nighttime dosing of alpha-adrenergic blocker, doxazosin: results from the halt study. Hypertension 2000; 35: 787-794.

42 Lusardi P, Mugellini A, Preti P, Zoppi A, Derosa G, Fogari R. Effects of a restricted sleep regimen on ambulatory blood pressure monitoring in normotensive subjects. Am J Hypertens 1996; 9: 503-505.
43 Miller MA, Cappuccio FP. Inflammation, sleep, obesity and cardiovascular disease. Curr Vasc Pharmacol 2007; 5: 93-102.

44 Wolk R, Somers VK. Sleep and the metabolic syndrome. Exp Physiol 2007; 92 : 67-78.

45 Goncharuk VD, van Heerikhuize J, Dai JP, Swaab DF, Buijs RM. Neuropeptide changes in the suprachiasmatic nucleus in primary hypertension indicate functional impairment of the biological clock. J Comp Neurol 2001; 431: 320-330.

46 Spiegel K, Leproult R, Van Cauter E. Impact of sleep debt on metabolic and endocrine function. Lancet 1999; 354: 1435-1439.

47 Spiegel K, Tasali E, Penev P, Van Cauter E. Brief communication: sleep curtailment in healthy young men is associated with decreased leptin levels, elevated ghrelin levels, and increased hunger and appetite. Ann Intern Med 2004; 141: 846-850.

48 Gallicchio L, Kalesan B. Sleep duration and mortality: a systematic review and metaanalysis. J Sleep Res 2009; 18: 148-158.

49 Grandner MA, Drummond SP. Who are the long sleepers? Towards an understanding of the mortality relationship. Sleep Med Rev 2007; 11: 341-360.

50 Bonnet MH, Arand DL. Hyperarousal and insomnia: state of the science. Sleep Med Rev 2010; 14: 9-15.

51 Bonnet MH. Evidence for the pathophysiology of insomnia. Sleep 2009; 32: 441-442.

(c) (i) (s) $\fallingdotseq$ This work is licensed under a Creative Commons Attribution-NonCommercial-NoDerivs 3.0 Unported License. To view a copy of this license, visit http://creativecommons. org/licenses/by-nc-nd/3.0/ 